\title{
La dermatite atopica: ieri, oggi e domani
}

\author{
Federica de Seta*, Luciano de Seta** \\ * Ospedale Santa Maria delle Grazie, Pozzuoli (Napoli); ** Pediatra, Napoli
}

leri

\section{Un po' di storia}

L'espressione "dermatite atopica" (DA), che deriva dal greco antico ó $\tau$ o $\pi \mathrm{o} \varsigma$, traducibile letteralmente come "senza un posto", fu introdotta da Arthur F. Coca e Robert Cooke nel 1923 e si riferiva alla mancanza di un posto nella classificazione medica allora in uso. Inoltre, designava un gruppo di affezioni caratterizzate da sensibilizzazione nei confronti di sostanze (allergeni) comunemente presenti nell'ambiente.

La dermatite atopica (DA) era già nota in tempi antichissimi, almeno 3-4.000 anni a.C. Gli antichi Egizi intorno al 1.500 a.C. riportavano nel famoso Papiro Ebers rimedi utili per combattere il prurito, uno dei sintomi più frequenti della DA. All'epoca dei Romani, il medico Celsus riferisce di una malattia detta "scabies" caratterizzata da indurimento della pelle con pustole secche o umide; mentre il poeta Svetonio descrive una malattia simile alla DA nell'imperatore Augusto. Così anche i Persiani e il medico Avicenna descrissero condizioni e trattamenti per una malattia simile alla DA.

I termini "allergia" e "atopia" furono introdotti ai primi del Novecento e nel 1933 la malattia venne per la prima volta definita "dermatite atopica" da Fred Wise e Marion Sulzberger. Approfondendo la componente allergica si scoprì che molti pazienti presentavano ipersensibilità ad allergeni e che restrizioni alimentari e ambientali mirate miglioravano la loro condizione. Uno degli alimenti "sotto accusa" fin dall'inizio è stato il latte per i neonati, per il quale sono state proposti diversi sostituti.

\section{Oggi}

\section{I sintomi}

"Dermatite" significa letteralmente processo infiammatorio della pelle. $\grave{E}$ quindi un termine generico, che si riferisce a varie condizioni, con caratteristiche e cause differenti e che può provocare diversi sintomi: arrossamento (eritema), prurito, desquamazione, comparsa di bollicine, comparsa di piccole lacerazioni della pelle.
La DA, anche detta eczema atopico (EA), è una malattia infiammatoria cronica della pelle che si manifesta di solito nei primi mesi di vita in circa il 15-20\% dei bambini, associata a una serie di sintomi e a un notevole disturbo della vita del bambino. Si presenta più frequentemente in bambini con familiarità per malattie allergiche tipo asma, eczema o rinocongiuntivite allergica.

È una malattia benigna, non infettiva né contagiosa. Il sintomo principale è il prurito: è presente in tutte le età, è intenso e quasi costante. La malattia nei primi mesi di vita si manifesta con un eritema più frequentemente al volto associato a prurito che il piccolo lattante manifesta piangendo e/o rotolandosi negli effetti letterecci (il guanciale, il lenzuolino ecc.). Altri sintomi sono: pelle secca o molto secca, placche rosse, essudazione, ispessimento della cute e lesioni da grattamento.

La DA nei neonati e nei lattanti compare più frequentemente sul viso. Verso i 5 anni, le lesioni sono più frequenti agli arti e al tronco, mentre, nei bambini più grandi, si localizzano tipicamente alle pieghe del gomito e delle ginocchia e attorno a occhi e bocca. Solitamente, oltre alla cute secca, possono presentarsi macchie ovali o rotonde, chiare o bianche, che vengono dette "pitiriasi alba". A volte, anche a causa delle piccole ferite che i bambini si procurano grattandosi, possono verificarsi delle sovra infezioni cutanee batteriche e la pelle può diventare ancora più umida o presentare zone con croste e pus (DA impetiginizzata). Come si vedrà appresso, esistono due tipi di DA: quella che compare nei primi mesi di vita e che può avere un'evoluzione verso la comparsa di una malattia allergica (asma, rinite), in genere già presente in uno o in entrambi i genitori; e una seconda forma che compare verso i 7-8 anni in figli di genitori non allergici (ma spesso portatori di DA) e che sovente si cronicizza richiedendo il ricorso a farmaci immunosoppressori. In un recente studio di coorte danese [1], molto complesso, per la prima volta si sono quantizzati i giorni di malattia e il numero di giorni del trattamento steroideo in bambini con DA nei primi tre anni di vita seguendo una popolazione di
700 bambini. I bambini con DA avevano una mediana di 136 giorni con sintomi (61-294 giorni) e 72 giorni in cui sono stati trattati con steroidi (27-145) durante i primi tre anni di vita, con il carico maggiore di malattia nel secondo anno di vita. L'analisi multivariata ha mostrato che la DA materna e la sensibilizzazione allergica infantile erano associate a un numero maggiore di giorni di DA, e che la DA materna e la mutazione della filaggrina, una delle cause più importanti nella patogenesi della sensibilizzazione allergica, erano associate a un numero maggiore di giorni di utilizzo di steroidi.

\section{L'epidemiologia}

La DA nei bambini si è diffusa in maniera esponenziale: i dati testimoniano che dagli anni Settanta è aumentata di 2-3 volte nei Paesi industrializzati. Colpisce attualmente circa il 10-20\% dei bambini e fino al 3\% degli adulti. La malattia si manifesta più comunemente entro i primi 5 anni d'età: il $60 \%$ dei bambini sviluppa la malattia entro il primo anno di vita e circa il 50\% entro i primi due anni di vita. Essa può precedere l'insorgenza di altre malattie allergiche, come l'asma allergico o la rinite allergica. I dati più accurati sulla diffusione mondiale della DA nei bambini sono stati forniti da un ormai vecchio studio internazionale: l'International Study of Asthma and Allergies in Childhood (ISAAC) [2] che si riferisce a quasi due milioni di bambini di ben cento Paesi. Lo studio ha messo in evidenza che in alcuni di essi più del $20 \%$ dei bambini soffre di dermatite atopica e che la sua diffusione varia notevolmente tra i vari Paesi. Per quanto riguarda i bambini di 6-7 anni, i dati indicano che la frequenza della malattia varia dallo $0,9 \%$ dell'India al 22,5\% dell'Ecuador, mentre per gli adolescenti di 13-14 anni la diffusione varia dallo $0,2 \%$ in Cina al 24,6\% in Colombia; sempre per i ragazzi di questa fascia d'età è stata osservata una frequenza del 15\% in 4 delle 9 aree geografiche indagate, tra cui l'Africa, l'Europa, l'America latina e l'Australia, con un forte aumento in America latina e nel Sudest asiatico.

Quanto alla situazione italiana, si conferma l'aumento della diffusione della DA. I 
dati sono stati estratti retrospettivamente dal database Pedianet (2006-2012) in bambini di età compresa tra 0 e 14 anni che presentavano una malattia della pelle a un controllo del loro pediatra di famiglia $(\mathrm{PF})$. Le stime di incidenza e prevalenza sono state calcolate per anno e stratificate per sesso, età e area geografica.

I risultati hanno mostrato una media di 145.233 bambini $(52,1 \%$ maschi) in cinque regioni italiane, registrati dai pediatri di famiglia, per un totale di 913.253 anni-persona di follow-up. La maggior parte dei pazienti proveniva dal nord-est (44,6\%) e il 37,7\% aveva un'età di 5-9 anni. Le stime di incidenza (nuovi casi/1.000 anni-persona) per la maggior parte delle malattie della pelle sono aumentate dal 2006 al 2012: la più alta crescita di casi è per la DA $(14,1$ contro 16,5$)$, l'orticaria acuta $(10,1$ contro 11,6$)$ e la dermatite da contatto $(9,3$ contro 10,8$)$ mentre la psoriasi è rimasta invariata nei 7 anni $(0,61$ vs 0,57$)$. Al contrario, le stime di prevalenza (casi/100 pazienti) sono aumentate da due a tre volte per diverse malattie della pelle, tra cui DA $(2,7 \%$ vs $8,5 \%)$, dermatite seborroica $(0,5 \%$ vs $1,6 \%)$, orticaria cronica $(0,4 \%$ vs $0,8 \%)$, e psoriasi $(0,09 \%$ vs $0,22 \%)$. Sono state osservate differenze di prevalenza in base alla fascia di età e all'area geografica per psoriasi, AD e orticaria [3]. Il consistente aumento della dermatite atopica tra i bambini e gli adolescenti pone la questione di un maggiore coinvolgimento dei pediatri per la diagnosi precoce e le cure della malattia.

\section{La patogenesi}

La cute costituisce il rivestimento più esterno del corpo umano, svolgendo l'essenziale funzione di barriera tra l'interno dell'organismo e l'ambiente esterno. Quest'attività è resa possibile da una varietà di meccanismi protettivi di tipo fisico, chimico e immunologico. Lo sviluppo della DA dipende dall'interazione tra fattori genetici e ambientali. L'associazione della DA con malattie allergiche e la presenza di esse nei genitori avevano indotto a pensare, fino a non molti anni fa, che la DA fosse causata da allergeni ambientali inalanti e alimentari in grado di indurre le lesioni caratteristiche della malattia. Di qui l'adozione di diete di eliminazione per cercare, anche sulla base dei risultati di prick positivi per trofoallergeni, di individuare la causa della DA. Era anche diffusa la convinzione che la DA, soprattutto quella del lattante e dei primi anni, fosse il primo step di una cosiddetta "marcia atopica" che però solo in alcuni casi progrediva verso le altre malattie allergiche respiratorie [4]. Questa convinzione ha portato a ritardare l'introduzione di nuovi alimenti nei bambini figli di genitori portatori di una malattia allergica. Il tutto con risultati molto contraddittori.

Sul finire degli anni Duemila un gruppo di ricercatori di diversi Paesi ha potuto correlare l'insorgenza della DA alla perdita di funzione di alcune varianti SNP (Single Nucleotid Polymorphism) del gene codificante per la filaggrina (FLG), una sostanza implicata nella costruzione dello strato corneo e quindi nella protezione, almeno in parte meccanica, del tegumento cutaneo [5-6]. In seguito a questa scoperta, la dermatite atopica ha assunto un nuovo aspetto patogenetico, per il quale, a un disordine primario della risposta immune come maggior candidato responsabile della malattia, si è venuta sostituendo l'idea di un difetto primario dei tegumenti.

Sulla base di questo e di successivi studi si è pervenuti alla conclusione che non sono gli allergeni alimentari o respiratori (risultati positivi in questi bambini e/o nei loro genitori atopici) la causa principale della malattia, ma la mancanza della FLG nella pelle che favorirebbe il passaggio di allergeni inalanti in soggetti predisposti geneticamente (atopici) e indurrebbe le lesioni della DA. Gli allergeni rappresentano una concausa della DA, tanto è vero che anche la terapia cortisonica e altri farmaci immunosoppressori migliorano nettamente la gran parte delle DA, soprattutto del bambino nei primi 2-3 anni di vita. La carenza geneticamente determinata della FLG induce un difetto di difesa dei tegumenti. Purtuttavia questa importante

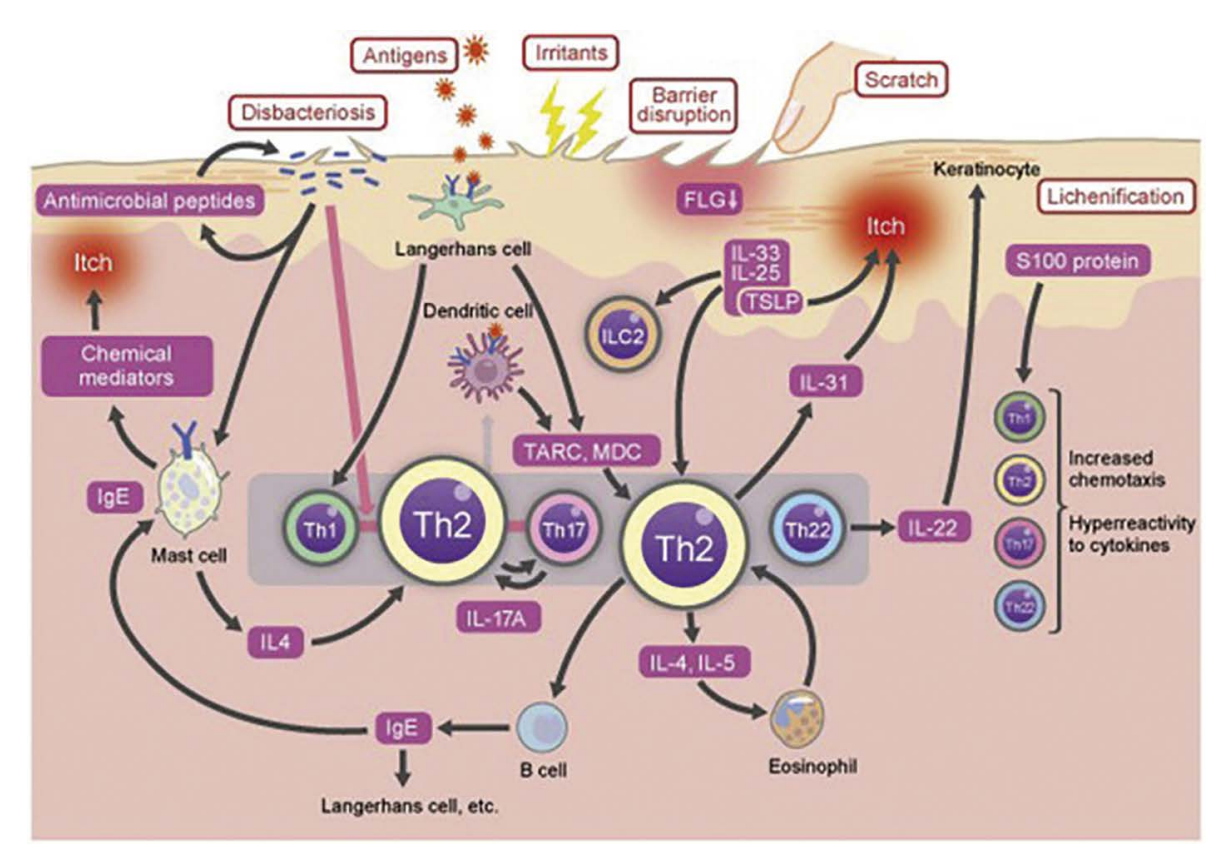

Figura 1. Patogenesi della dermatite atopica. FLG, filaggrin; IgE, immunoglobulin E; IL, interleukin; ILC, innate lymphoid cells; MDC, macrophage-derived chemokine; TARC, thymus and activation-regulated

chemokine; Th, T helper; TSLP, thymic stromal lymphopoietin. Da voce bibliografica [8]. anomalia genetica della cute non può da sola render conto di tutta l'eziopatogenesi della malattia, in particolare nei bambini nei quali la malattia compare fin dai primi mesi di vita e che hanno molte probabilità di andare incontro a malattie allergiche, segnatamente rinite e asma bronchiale allergico. La caratteristica che accomuna questi bambini è la propensione dei genitori a produrre anticorpi $\operatorname{IgE}$ verso antigeni ambientali comuni [7]. La DA è l'espressione fenotipica di un difetto di barriera cutanea geneticamente determinato. Questo perché, se è pur vero che il difetto genetico di barriera cutanea rappresenta la causa prima dell'eczema, è altrettanto vero che questo non è sufficiente da solo a spiegare tutta l'eziopatogenesi della confin dei primi mesi di vita, è strettamente collegata con le altre malattie "atopiche" dell'età successiva, la rinite allergica e l'asma bronchiale. Di fatto, come dimostrato in numerosi studi, i bambini con DA hanno un rischio tre volte maggiore di sviluppare asma e rinite allergica, se paragonati ai bambini senza eczema. Tuttavia, questo rischio quasi si azzera se vengono considerati i bambini con eczema iniziato dopo i 2 anni di età che sono anche quelli che non producono IgE verso allergeni comuni. Al contrario, nella DA dei primi mesi di vita, il rischio di sviluppare allergie verso alimenti e inalanti è molto alto: il $70 \%$ degli eczemi precoci e gravi svilupperà asma allergico entro i 6 anni, contro il $20-30 \%$ degli eczemi lievi e 1'8\% della popolazione non selezionata. Questo per sottolineare dizione che, quando interessa il bambino 
che la "dermatite atopica" precoce è diversa dalla DA del bambino più grandicello, non associata alla predisposizione "atopica”, ma anche per richiamare il concetto che il difetto di barriera cutanea non è da solo sufficiente a spiegare i diversi fenotipi dell'eczema infantile. In altre parole, pur ricordando che i meccanismi alla base delle DA sono genetici e ambientali e non del tutto chiariti in modo definitivo, bisogna sottolineare che non è l'allergia da sola la causa della DA. È ipotizzabile che una parte sola delle DA infantili si associ a una sensibilizzazione allergica per allergeni alimentari o respiratori e che solo questi possano evolvere in asma bronchiale [7] (Figura 1).

Una diminuzione della funzione di barriera cutanea può consentire agli allergeni di penetrare facilmente nella pelle. Gli allergeni, che sono molecole estranee, vengono eliminati dall'immunizzazione e dalle reazioni allergiche. Gli allergeni, quali l'acaro della polvere e gli allergeni proteici, inducono reazioni immunitarie di tipo 2 attraverso l'attività della proteasi. Le cellule $\mathrm{T}$ helper possono essere distinte in cellule Th1 e Th2. È stato dimostrato che le cellule Th1 sono coinvolte nell'immunità cellulo-mediata, mentre le cellule Th2 sono principalmente associate a reazioni allergiche. L'interleuchina (IL)-33, IL-25 e la linfopoietina stromale timica (TSLP) prodotte dai cheratinociti epidermici sono associate alla migrazione delle cellule Th2 alla lesione. La risposta immunitaria di tipo 2 porta all'induzione di anticorpi IgE allergene-specifici. Le cellule di Langerhans e i mastociti esprimono il recettore $\mathrm{IgE}$ ad alta affinità (FceRI) e rilasciano citochine e trasmettitori chimici (per esempio istamina) attraverso il legame di IgE allergene-specifiche per indurre l'infiammazione. Le cellule Th22 producono IL-22 dopo la migrazione sulla pelle, probabilmente attraverso la regolazione da parte delle cellule dendritiche cutanee attivate, che induce acantosi epidermica. La proteina S100 prodotta dal danno epidermico attiva ulteriormente i linfociti [8].

\section{Domani}

Terapia

Anche sulla scorta di quanto abbiamo fino a oggi appresso sulla patogenesi della DA, si è ipotizzato che l'applicazione dalla nascita di creme emollienti o creme base sulla pelle di bambini geneticamente predisposti avrebbe potuto prevenire la comparsa della DA e delle eventuali successive malattie allergiche ricostituendo in parte la carente filaggrina. Alcune ricerche comparse in letteratura abbastanza recentemente [9-10] non hanno dimostrato che l'incidenza di DA in bambini trattati dalla nascita con terapia emolliente topica fosse inferiore a quelli dei bambini non trattati. Quindi le creme emollienti si sono dimostrate, senza ombra di dubbio, del tutto inefficaci nel prevenire o guarire la DA. Ciò non toglie che queste creme possano essere di grande aiuto anche sulla pelle trattata con steroidi o altri farmaci e possono contribuire notevolmente a ridurre l'impiego di altri farmaci [9].

Quindi l'applicazione di una crema base non può restituire l'integrità alla cute lesa dalla carenza di filaggrina. Che in parte può essere ricostituita dai cortisonici topici utilizzati a cicli terapeutici o alla riaccensione della malattia, confermandosi ancora una volta il farmaco proattivo più efficace in prima battuta. Gli attuali trattamenti cardine con steroidi proattivi, e, nei casi più resistenti, con gli inibitori della calcioneurina, riescono a risolvere la gran parte degli eczemi, soprattutto dei bambini nei primi tre anni di vita.

Le linee guida giapponesi del 2020 [8] sottolineano i tre aspetti fondamentali che devono guidare il pediatra nel trattamento della DA:

1 l'uso proattivo dei cortisonici topici;

2 l'impiego di creme barriera per cercare di arginare i danni dovuti al difetto di barriera (questa dovrebbe migliorare la barriera cutanea e in genere sono creme a base di paraffina, glicerina e altri eccipienti);

3 offrire ai genitori tutte le spiegazioni sulla natura della malattia, sulla sua evoluzione e sui principi del trattamento.

Sempre maggiore spazio occupano in letteratura gli studi che confermano l'utilizzo degli anticorpi monoclonali, come il Dupolimumab [11], un anticorpo monoclonale contro la parte condivisa del recettore dell'IL-4 e dell'IL-13. Recentemente è stato proposto il Baricitinib [12], un inibitore del recettore di membrana JAK 4, coinvolto nell'attivazione della cascata citochimica. Tutti questi nuovi farmaci hanno dimostrato una consolidata validità nei casi più gravi e resistenti alla terapia corticosteroidea topica.

La pediatra o il pediatra hanno a disposizione un bagaglio non indifferente di farmaci eziologici e coadiuvanti che possono, se scelti con accuratezza, dare ottimi risul- tati. I pediatri dovranno però stabilire con i genitori un ottimo rapporto che sarà indispensabile per far nascere e crescere la fiducia nella guarigione o in un accettabile miglioramento.

fedeseta@gmail.com

1. Ruge IF, Thorsteinsdóttir S, Nørgaard S, et al. Symptom burden of atopic dermatitis in early childhood assessed from daily monitoring of symptoms and topical steroid use. J Am Acad Dermatol. 2021 Mar;84(3):725-734.

2. Worldwide variation in prevalence of symptoms of asthma, allergic rhinoconjunctivitis, and atopic eczema: ISAAC. The International Study of Asthma and Allergies in Childhood (ISAAC) Steering Committee. Lancet. 1998 Apr 25;351(9111):1225-1232.

3. Cantarutti A, Donà D, Visentin F, et al. Epidemiology of Frequently Occurring Skin Diseases in Italian Children from 2006 to 2012: A Retrospective, Population-Based Study. Pediatr Dermatol. Sep-Oct 2015;32(5):668-678. 4. Bantz SK, Zhu Z, Zheng T. The Atopic March: Progression from Atopic Dermatitis to Allergic Rhinitis and Asthma. J Clin Cell Immunol. 2014 Apr;5(2):202.

5. Bisgard H, Simpson A, N.,Palme CN, et al. Gene-environment interaction in the onset of eczema in infancy Filaggrin loss of function mutations enhanced by neonatal cat exposure. PLoS Med 2008; 5;e131 doi: 10.1371/journal. pmed.0050131.

6. Irvine AD, McLean WH, Leung DY. Filaggrin mutations associated with skin and allergic diseases. N Engl J Med. 2011 Oct 6;365(14):1315-1327.

7. Longo G. Dermatite atopica: intendiamoci sulle parole. Medico e Bambino. 2015;34(6):347350.

8. Horimukai K, Morita K, Narita M, et al. Application of moisturizer to neonates prevents development of atopic dermatitis. J Allergy Clin Immunol. 2014 Oct;134(4):824830.e6.

9. Damiani G, Calzavara-Pinton P, Stingeni $\mathrm{L}$, et al. Italian guidelines for therapy of atopic dermatitis-Adapted from consensus-based $\mathrm{Eu}-$ ropean guidelines for treatment of atopic eczema (atopic dermatitis). Dermatol Ther. 2019 Nov;32(6):e13121

10. Katoh N, Ohya Y, Ikeda M, et al. Japanese guidelines for atopic dermatitis 2020. Allergol Int. 2020 Jul;69(3):356-369.

11. Paller AS, Siegfried EC, Thaçi D, et al. Efficacy and safety of dupilumab with concomitant topical corticosteroids in children 6 to 11 years old with severe atopic dermatitis: A randomized, double-blinded, placebo-controlled phase 3 trial. J Am Acad Dermatol. 2020 Nov;83(5):1282-1293.

12. Simpson EL, Lacour J-P, Spelman L, et al. Baricitinib in patients with moderate-to-severe atopic dermatitis and inadequate response to topical corticosteroids: results from two randomized monotherapy phase III trials. Br J Dermatol. 2020 Aug;183(2):242-255. 